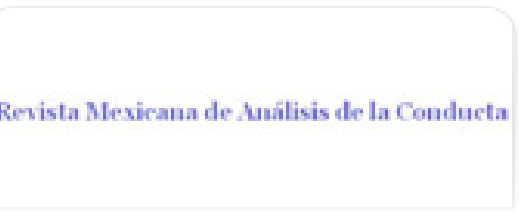

Revista Mexicana de Análisis de la Conducta ISSN: 0185-4534

editora@rmac-mx.org

Sociedad Mexicana de Análisis de la Conducta

México

MORALES CHAINÉ, SILVIA; SANTOYO VELASCO, CARLOS

INCREMENTO EN LA RESISTENCIA AL CAMBIO POR REFORZAMIENTO DE CONDUCTA ALTERNATIVA: UN ESTUDIO TRASLACIONAL

Revista Mexicana de Análisis de la Conducta, vol. 40, núm. 1, junio, 2014, pp. 25-50

Sociedad Mexicana de Análisis de la Conducta

Distrito Federal, México

Disponible en: http://www.redalyc.org/articulo.oa?id=59335810002

Cómo citar el artículo

- Número completo

- Más información del artículo

- Página de la revista en redalyc.org

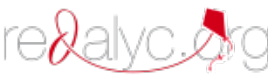

Sistema de Información Científica

Red de Revistas Científicas de América Latina, el Caribe, España y Portugal

Proyecto académico sin fines de lucro, desarrollado bajo la iniciativa de acceso abierto 


\title{
INCREMENTO EN LA RESISTENCIA AL CAMBIO POR REFORZAMIENTO DE CONDUCTA ALTERNATIVA: UN ESTUDIO TRASLACIONAL
}

\author{
INCREASED RESISTANCE TO CHANGE FOLLOWING \\ DIFFERENTIAL REINFORCEMENT OF ALTERNATIVE BEHAVIOR: \\ A TRANSLATIONAL STUDY \\ SILVIA MORALES CHAINÉ Y CARLOS SANTOYO VELASCO \\ UNIVERSIDAD NACIONAL AUTÓNOMA DE MÉXICO
}

\section{RESUMEN}

La investigación traslacional tiene como fin alcanzar implicaciones prácticas a partir de los hallazgos derivados de la investigación básica. El objetivo del estudio fue describir las variables que determinan la resistencia al cambio empleando una tarea de unión de rompecabezas en una computadora. Participaron cuatro niños entre ocho y nueve años y se empleó un diseño de reversión $\mathrm{ABAC}$, donde $\mathrm{A}$ correspondió a la línea base y $\mathrm{B}$ y $\mathrm{C}$ a las pruebas de resistencia al cambio: entrega no contingente del reforzador y extinción, respectivamente. En cada condición experimental se utilizó un programa múltiple de tres componentes. Cada componente duraba $60 \mathrm{~s}$ y se señaló diferencialmente mediante el color del fondo de la pantalla. En cada componente estuvo vigente un programa concurrente: extinción (EXT)- intervalo variable (IV) 15, EXT- IV60 y reforzamiento diferencial de conducta alternativa (RDA) mediante un IV20 IV60. Cada lado de la pantalla se asoció con el programa de extinción o el programa de intervalo variable señalado. Durante los tres componentes, juntar las piezas del rompecabezas se reforzó con puntos que podían intercambiarse por comida o juguetes. Se encontró que la tasa de respuesta mantenida por el programa IV fue baja cuando hubo reforzamiento simultáneo a la respuesta alternativa del lado izquierdo, pero fue más resistente al cambio. La relación estímulo-reforzador establecida determinó parcialmente la resistencia al cambio. Emplear un RDA resulta en 
una disminución de la conducta meta durante la línea base, pero aumenta la resistencia al cambio.

Palabras clave: Resistencia al cambio, reforzamiento diferencial de conducta alternativa, psicología traslacional

\begin{abstract}
Translational research aims to accomplish applied gains through basic research. The purpose of this study was to describe the determinants of resistance to change on a task that consisted of joining two puzzle pieces on a computer screen. The experiment was conducted with four children, and an ABAC design was used, in which $A$ was the baseline and $B$ and $C$ were the resistance to change tests: non contingent reinforcement and extinction, respectively. During each experimental condition, a three-component multiple schedule was in effect. Each component lasted for 60 seconds and was signaled differentially by the background color of the screen. Within the three components, one of the following concurrent schedules was in effect: extinction (EXT) -variable interval (VI) $15 \mathrm{~s}$, EXT -VI $60 \mathrm{~s}$, or a concurrent VI $20 \mathrm{~s}-\mathrm{VI}$ $60 \mathrm{~s}$, which represented a differential reinforcement of alternative behavior (DRA) arrangement. Each side of the screen was associated with the described extinction or variable interval schedules. During the three components, joining the puzzles was reinforced with points that could be exchanged for food or toys. Response rates during the VI $60 \mathrm{~s}$ schedule were the lowest when the VI $20 \mathrm{~s}$ was presented concurrently on the left side of the screen, but responding in this option was more resistant to change. Resistance to change was partially determined by the established stimulusreinforcer contingencies. DRA decreases the frequency of target behavior, but increases its resistance to change.

Keywords: resistance to change, differential reinforcement of alternative behavior, translational research

La investigación traslacional tiene como fin alcanzar implicaciones prácticas a partir de los hallazgos derivados de la ciencia básica. Así, la solución exitosa de problemas sociales debiera resultar de la aplicación de procedimientos de intervención derivados de los principios básicos que permiten comprender la fuerza de la conducta (Morales \& Santoyo, 2012). La traslación implica una secuencia de eventos, desde el descubrimiento de conocimiento nuevo hasta su adopción y uso en escenarios naturales de la vida cotidiana, para lograr el bienestar social (Santoyo, 2012; Shull \& Lawrence, 1998). La labor de translación incorpora diversas actividades estratégicas y metodológicas para poner a prueba los hallazgos de la ciencia básica en contextos nuevos y bajo restricciones cada vez más naturales (Santoyo, 2012).
\end{abstract}


De acuerdo con Buskist, Morgan y Terrel (1985), en el campo del análisis experimental de la conducta, se deben diseñar procedimientos para realizar investigación operante con humanos, preservando y extendiendo su núcleo conceptual e innovando estrategias metodológicas, así como contribuir a entender las limitaciones de las explicaciones, analizando los contextos en los que estas son aplicables y aquellos en los que dejan de serlo (McGuire, 1997; Santoyo, 2012).

En este ámbito, la propuesta sobre la teoría de momentum conductual (Nevin, 1974; Nevin \& Shahan, 2011) ha centrado el interés en el estudio de la resistencia al cambio de la conducta operante y en su relevancia para la reducción o eliminación de las conductas problema así como para el establecimiento de conducta adaptativa permanente. El estudio de la fuerza de una respuesta se ha centrado en la evaluación de la correlación entre la tasa relativa de ocurrencia y la tasa relativa de reforzamiento (Herrnstein, 1970). La teoría de momentum conductual aborda el concepto de fuerza de respuesta en términos de su resistencia al cambio en el contexto original de adquisición (Nevin, 1974, 1979, 1996, Nevin, Tota, Torquato, \& Shull, 1990; Nevin \& Grace, 2000; Nevin \& Shanan, 2011). La resistencia al cambio puede ser calificada como relativamente baja cuando se observa que el patrón de respuesta cambia rápidamente; o relativamente alta cuando las tasas de respuesta cambian lentamente, ante la modificación en las condiciones del contexto de adquisición (e.g., en las contingencias de reforzamiento) o en aquellas relacionadas con la motivación del individuo, como es el caso de la saciedad (Nevin, Mandell, \& Atak, 1983).

Nevin et al. (1990) propusieron que la conducta reforzada frecuentemente se vuelve más resistente al cambio y que este incremento en su resistencia no necesariamente implica un incremento en la tasa de conducta. Sin embargo, los efectos del fortalecimiento de la respuesta pueden hacerse evidentes cuando el responder es interrumpido. Nevin et al. (1990) entrenaron a pichones a responder a dos programas de intervalo variable (IV) idénticos en los dos componentes de un programa múltiple y agregaron reforzamiento independiente a la respuesta de acuerdo a un programa de tiempo variable (TV) en uno de los componentes. Sus resultados mostraron que la conducta que recibió reforzamiento adicional no contingente mostró mayor resistencia al cambio que la conducta que se mantenía por los mismos programas de reforzamiento, pero que no recibía reforzamiento adicional no contingente. Los autores concluyeron que la resistencia al cambio depende directamente de la tasa o cantidad de reforzamiento señalado por un estimulo (relación estímulo reforzador) y no de si todos los reforzadores son contingentes a la respuesta meta.

La literatura básica reporta tres clases de hallazgos para la comprobación de la teoría de la resistencia al cambio (Nevin et al., 1990): 1) La tasa de respuesta está determinada por la relación respuesta-reforzador, ya que la tasa de respuesta es mayor en los componentes donde existe mayor tasa de reforzamiento y es menor en los 
componentes con reforzadores adicionales; 2) La resistencia al cambio está determinada por la relación estímulo-reforzador (Mandell, 2000), al encontrar que dicha resistencia es mayor en los componentes asociados con altas tasas de reforzamiento y en los componentes con reforzadores adicionales no contingentes o contingentes a una respuesta alternativa (Nevin \& Shahan, 2011); y 3) La relación respuesta-reforzador, que determina la tasa de respuesta, es independiente de la relación estímulo-reforzador, que determina la resistencia al cambio (Nevin \& Grace, 2000). Así, la resistencia al cambio debería ser igual en dos componentes con tasas globales de reforzamiento similares, aún cuando tales conductas meta reciban tasas diferenciales de reforzamiento relativo durante la línea base.

Diversos estudios han intentado llevar a cabo la traslación de los hallazgos relacionados con la teoría de resistencia al cambio (Roane, Kelley, Trosclair, \& Hauer, 2004; Parry-Cruwys, Neal, Ahearn, Wheeler, Presmchander, Loeb, \& Dube, 2011) entre especies y con diferentes programas de reforzamiento (Cohen et al., 1993; Harper, 1999; Mandell, 2000; Mauro \& Mace, 1996; Mace et al., 2010; Nevin \& Shahan, 2011). Así, Houlihan y Brandon (1996) y Nevin (1996) indicaron que la investigación en este campo debe resolver tres limitantes importantes. Primero, es necesario que el estudio de la resistencia al cambio se lleve a cabo sobre la tasa asintótica de una operante discriminada; Segundo, las pruebas para evaluar la resistencia al cambio deben planearse como fases independientes de las contingencias de adquisición y mantenimiento de la conducta; por ello, es importante utilizar diseños intra-sujeto. Tercero, las pruebas de resistencia al cambio deben presentarse por periodos breves de tiempo para reducir los efectos a largo plazo de la interacción entre la prueba de resistencia y la línea base, para identificar las variables determinantes y controlar la historia de reforzamiento.

A partir de las recomendaciones para los estudios que buscan la traslación de las premisas de la teoría de la resistencia al cambio con humanos, Mace et al., (1990) y Morales y Santoyo $(2005,2012)$ Ilevaron a cabo réplicas sistemáticas de los estudios de Nevin et al. (1990) con humanos con y sin dificultades académicas o de desarrollo, respectivamente. Mace et al. (1990) evaluaron la resistencia al cambio con adultos diagnosticados con retraso mental severo. Para ello midieron la conducta de clasificar 40 objetos de cocina conforme a su color (verde o rojo) colocándolos en un contenedor. Expusieron a los participantes a un programa múltiple concurrente IV60s para ambos colores, pero se agregó un programa de tiempo variable TV30s durante el componente asociado con los objetos rojos; mantuvieron las condiciones de reforzamiento de la línea base mientras utilizaban como disruptor un programa de entretenimiento en una televisión durante todo el componente. Los resultados mostraron que la tasa de respuesta, durante la línea base fue baja en el componente con reforzadores adicionales y que la resistencia al cambio fue mayor en los componentes asociados con 
altas tasas de reforzamiento y en aquellos con reforzadores adicionales no contingentes a la respuesta.

En términos particulares del reforzamiento diferencial a conducta alternativa, Morales y Santoyo (2012), trabajaron con una conducta académica a través de dos pruebas de resistencia al cambio: extinción y la entrega no contingente del reforzador con niños. Utilizaron un programa múltiple concurrente con estímulos específicos asociados: EXT-IV15 con fondo blanco, EXT IV60 con fondo rojo e IV20 IV60 con fondo verde, asegurando el reforzamiento global similar en el primer y el tercer componentes. Se presentaron sumas de dos dígitos en hojas de papel del lado derecho e izquierdo del participante, asociados a los tres diferentes colores y cada lado se asoció con el programa de extinción o el programa de intervalo variable señalado. En el estudio se encontró una tasa menor de respuesta cuando existía reforzamiento a una respuesta alternativa y que la relación estímulo-reforzador determinó la resistencia al cambio al obtener una mayor persistencia de la respuesta cuando hubo reforzamiento adicional a una respuesta alternativa. Estos datos son consistentes con los de Mace et al. (1990) en cuanto a que las proporciones de respuestas respecto a la línea base dependen de las tasas de reforzamiento y no de las tasas de respuesta durante la misma línea base. Sin embargo, Morales y Santoyo (2012) no encontraron independencia entre estos determinantes, señalando que en el trabajo con humanos debe mantenerse el control en la historia previa a la línea base y registrar diferentes topografías de respuesta, debido a que la resistencia al cambio con humanos puede depender, también, del reforzamiento diferencial que se presenta a otras conductas presentes al mismo tiempo

Recientemente, Nevin y Shahan (2011) analizaron diversos estudios para comprender la traslación de las premisas de momentum encontrando que el efecto de la extinción como disruptor depende no solo del retiro de las contingencias entre la respuesta y el reforzador, sino también al decremento en la generalización de los estímulos y de su combinación con el retiro del refozamiento, con el paso del tiempo. En un análisis exhaustivo del modelo de resistencia, Nevin y Shahan concluyeron que la extinción ocurre rápidamente cuando la omisión del reforzador es altamente notoria (efecto parcial de extinción, PREE por sus siglas en ingles). Pero, indicaron que la curva de extinción se revierte cuando la omisión del reforzador es menos evidente. El efecto de la suspensión de la contingencia también es poco perceptible cuando la tasa de respuesta no tiene consecuencias y los reforzadores se otorgan de manera independiente a la respuesta en la misma tasa que en el entrenamiento. Nevin et al. (1990) demostraron que la resistencia a la extinción de la conducta meta fue evidentemente mayor cuando se expresaron los datos como proporciones de la línea base.

Nevin y Shahan (2011) señalaron que el reforzamiento diferencial de conducta alternativa (RDA) aumenta la resistencia a la extinción de la conducta meta porque 
aumenta la tasa global del reforzamiento obtenido en el contexto de los estímulos discriminativos. Mace et al. (2010) también usaron contingencias RDA para reducir conductas problema. Encontraron que las conductas de jalar el cabello, robar alimentos o de agresión disminuyeron más rápidamente y a un nivel más bajo cuando se aplicó extinción directamente después de la línea base que cuando se aplicó extinción después del tratamiento con RDA a juego apropiado y peticiones adecuadas de alimento. Los autores concluyeron que aunque el RDA disminuye la tasa de la conducta problema, también incrementa su resistencia a la extinción. Específicamente, la tasa de la conducta problema puede ser incluso más alta si es reducida por RDA y extinguida que si no se usa RDA antes de su extinción. Así, las intervenciones que utilizan RDA para reducir conducta problema pueden resultar contra-producentes.

Por lo tanto, con el fin de evaluar la traslación de los hallazgos de la teoría de resistencia al cambio en un escenario con control experimental pero con características similares a los escenarios naturales del niño (Santoyo et al., 2007) y de analizar los efectos del RDA sobre la resistencia al cambio de la conducta meta, el presente estudio tuvo como objetivo describir las variables que determinan la resistencia al cambio de una tarea a realizarse en la computadora con niños en edad escolar a través de un programa múltiple con tres programas concurrentes, en una réplica sistemática del Experimento 2 diseñado por Nevin et al. (1990).

\section{Método}

\section{Participantes}

Los participantes fueron cuatro niños, seleccionados por cuota, sin problemas académicos ni de conducta. El participante $S 1$ tenía 9 años de edad y cursaba el cuarto año de primaria. Las participantes S2, S3 y S4 tenían 8 años de edad y cursaban el tercer año. Todos eran hijos del personal administrativo de una institución de educación superior de la Ciudad de México y asistían diariamente con sus padres a la institución. En todos los casos se contó con la autorización expresa de los niños y sus padres. Los niños trabajaron en un cubículo de $3 \times 2$ metros equipado con una mesa, una silla y una computadora de escritorio.

\section{Aparatos}

Se utilizó una computadora DELL ${ }^{\circledR}$ Modelo Pentium IV equipada con el programa Visual Basic Runtime Files 6.0 SP5 ${ }^{\circledR}$ para Windows ${ }^{\circledR} X \mathrm{X}$, a partir del cual y con base en la formula de progresión de Fleshler y Hoffman (1962) se programaron los valores de los componentes de intervalo variable (IV) del programa múltiple concurrente para 


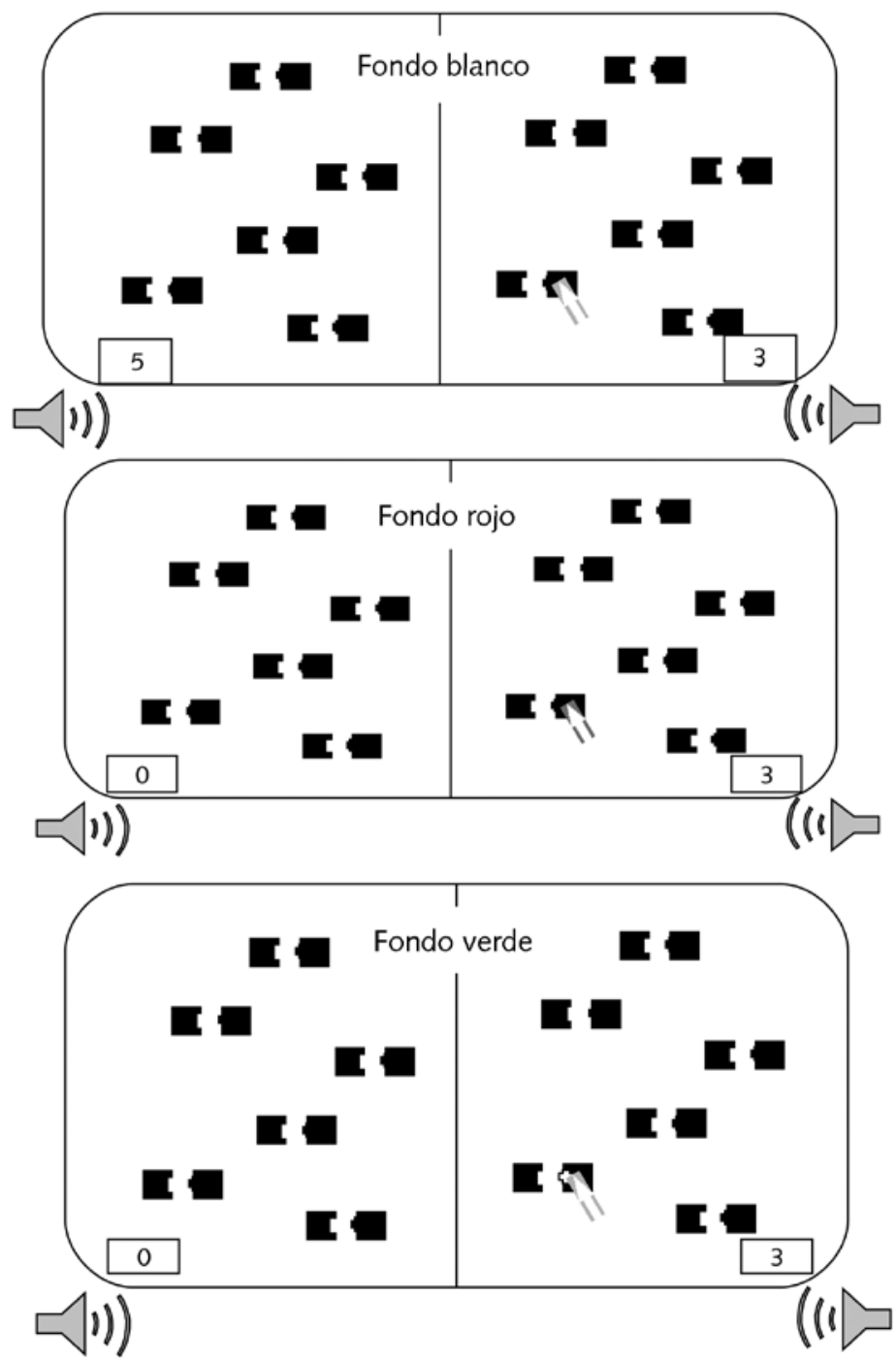

Figura 1. Representación gráfica de la respuesta de armar rompecabezas de dos piezas, presentada en la pantalla de la computadora. Cada componente duraba un minuto y el tiempo entre componentes fue de 5 segundos 
cada sesión. Siempre se mostraron seis rompecabezas de dos piezas, no alineados, en cada lado, programados para separarse uno o dos segundos después de haber sido unidos al presionar el botón del ratón, de tal manera que continuamente existía la oportunidad de unir rompecabezas. Cada componente del programa presentaba dos cuadros, uno de cada lado en la base de la pantalla, donde se acumulaban los puntos obtenidos. Los puntos se presentaban acompañados de un tono y se otorgaban de manera contingente a la respuesta de acuerdo a los diferentes programas de Intervalo Variable (IV) y asociados a uno de tres colores: blanco, rojo o verde. El programa registraba la frecuencia y duración de las respuestas, la duración de los intervalos, los puntos obtenidos, así como el tiempo en el que se proporcionaban (ver Figura 1).

\section{Procedimiento}

Se utilizaron diseños de reversión $\mathrm{ABAC}$, donde $\mathrm{A}$ correspondió a la fase de Línea Base (LB) y $\mathrm{B}$ o $\mathrm{C}$ constituyeron las pruebas de resistencia al cambio basadas en la entrega no contingente del reforzador (NC) y en la extinción (EXT), respectivamente. Se contrabalanceó la presentación de las pruebas de resistencia. Esto es, para los participantes uno y dos se presentó primero la entrega NC y para los niños tres y cuatro, la EXT. En la segunda fase de prueba se invirtió la presentación de las pruebas de resistencia al cambio.

Previo a la LB, se contó con un Muestreo de reforzadores y un entrenamiento preliminar de la conducta meta: unir rompecabezas de dos piezas presionando el botón izquierdo del mouse de la computadora.

Muestreo de reforzadores. Se llevó a cabo, con cada niño por separado, el método de evaluación de la preferencia individual de reforzadores "elección por pares" (Fisher \& Mazur, 1997). Se colocó a cada niño frente a una mesa con 36 pares de estímulos, tanto consumibles (e. g., cacahuates, chocolates, galletas) como manipulables (juguetes armables, carritos hot wheels $₫$, rompecabezas, etc.), obtenidos a partir de una lista con nueve peticiones individuales y se les pidió que eligieran uno de ellos diciendo: "Elige el que más te guste y tómalo".

Cada uno de los reforzadores fue asociado con los demás durante un ensayo de pares por sesión y se registraron las preferencias para cada niño. Una vez determinadas las preferencias se procedió a establecer el costo de intercambio diseñando una jerarquía de reforzadores y asignando el mayor costo a aquellos reforzadores preferidos y el costo menor a los menos preferidos, por participante (Morales \& Santoyo, 2012).

Condiciones constantes durante el entrenamiento preliminar, LB y pruebas de resistencia al cambio. Durante estas fases, la estrategia del programa múltiple de tres componentes con programas concurrentes asociados a un color particular, el formato de presentación de los rompecabezas de dos piezas en la pantalla dividida en dos, 
la duración de cada sesión (33 minutos) y la forma en que se colocaba la experimentadora durante la sesión, fueron similares.

En cada sesión, la experimentadora se colocaba detrás del niño, abría el programa Visual Basic, presentaba las instrucciones, permitiendo dejar a la vista la pantalla y el mouse y proporcionaba fichas de colores, una por cada punto ganado acorde al IV designado, colocándolas en el lado correspondiente y del color del componente, sin exceder cinco segundos después de que el programa entregaba el punto (acompañado de un sonido) programado por la realización de la conducta meta de acuerdo al valor del intervalo. Cada componente del programa múltiple duraba 60 segundos, seguido de un periodo de cinco segundos entre componentes (cambio de color de la pantalla) y cada sesión diaria concluía cuando todos los componentes se presentaban nueve veces. Al final de cada sesión, el niño cambiaba las fichas por los reforzadores elegidos.

Durante las sesiones, se colocó a cada niño frente a la pantalla y el mouse y se le proporcionaron verbalmente las siguientes instrucciones:

—Nombre del niño—, "A continuación se te presentará una serie de rompecabezas que puedes unir con solo apretar el botón izquierdo del ratón. Como podrás ver, la pantalla frente a ti está dividida en dos. Tú puedes decidir de qué lado quieras trabajar, puedes decidir cuando quieres trabajar y también puedes decidir cuántos rompecabezas quieres armar en cada lado. No tienes que hacer todos los rompecabezas, observa cómo funciona el programa y gana fichas. Cada vez que ganes una ficha yo la colocaré del lado que la ganaste y del color que la ganaste para que puedas cambiarlas por lo que te guste de la tiendita al final del programa. ¿Estás listo (a)? Puedes empezar".

Entrenamiento preliminar. Durante el programa múltiple, durante diez sesiones se entrenó a los participantes (en la Tabla 1 se especifica el orden de la condición y su número de sesiones programadas), variando la tasa relativa de reforzamiento en cada componente y de cada lado de forma sistemática para asegurar que la respuesta de unir rompecabezas cambiaba de acuerdo con dichas tasas y que no se desarrollaban tendencias o preferencias hacia alguno de los lados de la pantalla.

Línea base (LB). Durante un mínimo de 10 sesiones, en los primeros dos componentes del programa múltiple se presentó un programa de IV y uno asociado con extinción, mientras que en el tercer componente operaba un programa concurrente IV20 IV60 RDA (Nevin \& Shahan, 2011; Ver Tabla 1).

Para el criterio de estabilidad en LB se estableció, además del número mínimo de sesiones, que la tendencia de la tasa de respuesta no variara más del $15 \%$, en por lo menos dos de los tres componentes (Morales \& Santoyo, 2012). 
MORALES CHAINÉ Y SANTOYO VELASCO

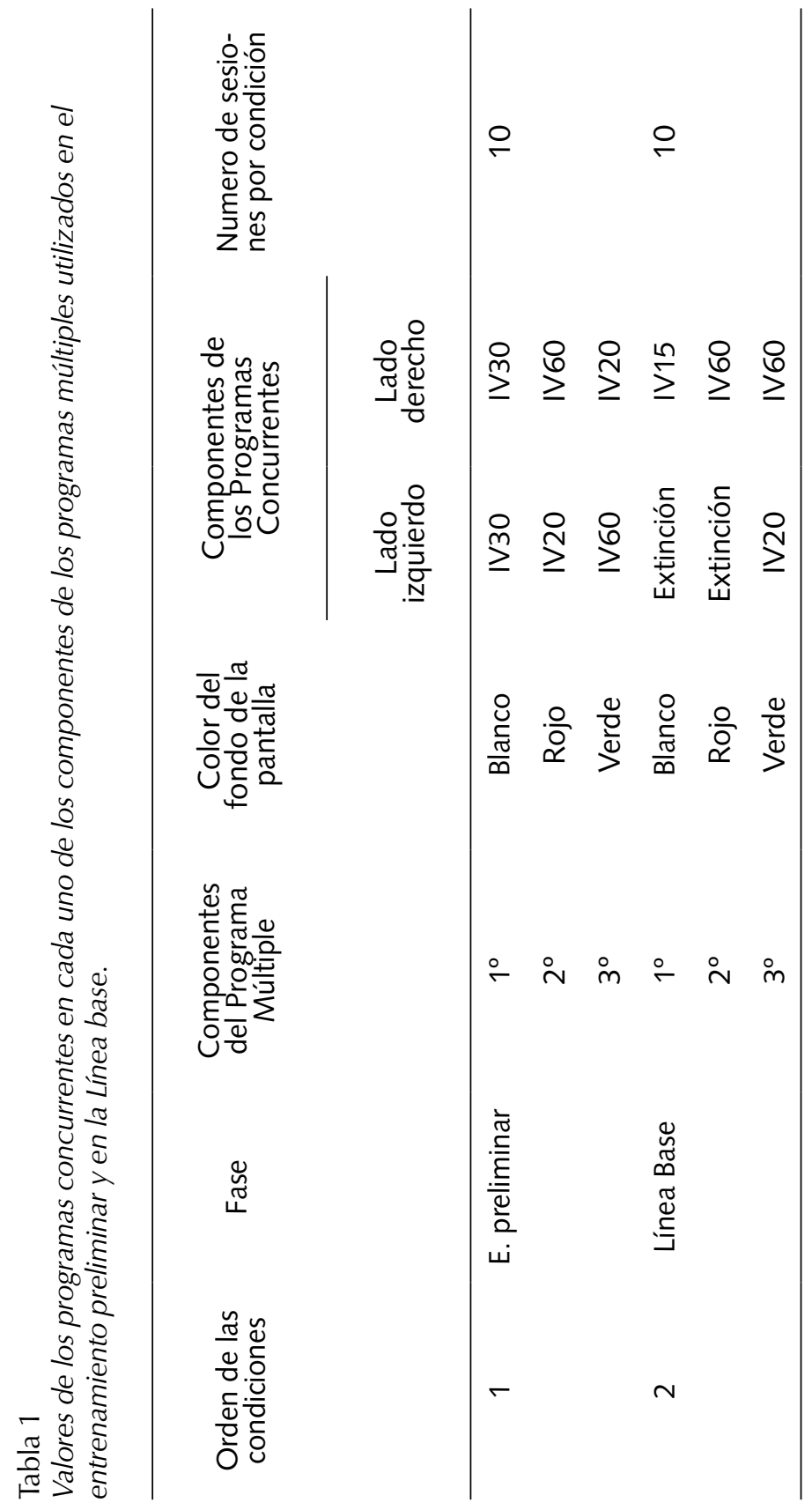


Entrega no contingente del reforzador (NC). Esta condición consistió de tres sesiones. Las condiciones del procedimiento general fueron similares a las fases de línea base, solo que durante estas se realizó la entrega de fichas (programadas por cada componente) previo al inicio de la sesión como procedimiento análogo al prealimento (saciedad, Nevin et al., 1990).

Extinción (EXT). La condición de extinción consistió de tres sesiones, de tal manera que se cumplieran los requerimientos de presentar la prueba de resistencia al cambio en pocas sesiones para minimizar los efectos a largo plazo de la interacción entre la prueba de resistencia y las condiciones de reforzamiento de línea base (Nevin, 1996). Las condiciones del procedimiento fueron similares a las de línea base, en cuanto a que los participantes podían unir rompecabezas en ambos lados de acuerdo a la tarea, excepto que no se entregaban los puntos (y por lo tanto ni los sonidos, ni fichas previamente asociadas).

Al final de cada estudio se aplicó un cuestionario de satisfacción e identificación de contingencias, donde se leía un grupo de preguntas al niño y, mientras grababa en formato de audio, registraba las respuestas en el cuestionario. Se le preguntaba al niño sí sabía cómo funcionaba el programa, si había diferencias en cómo funcionaba el programa de acuerdo al color de la pantalla, en que color ganaba más fichas, cual era la regla para ganar fichas en cada color y cuantas fichas ganaba en cada lado y de cada color. Con ello se buscaba tener un indicador de la sensibilidad del niño a las contingencias de reforzamiento en los programas de intervalo variable (Weiner, 1962).

Para la evaluación de la resistencia al cambio se obtuvieron razones de cambio a partir de la ecuación 1, derivada de las evaluaciones de resistencia al cambio propuestas por Nevin y Grace (2000):

De aquí que R EXT resulta de la obtención de la tasa de respuesta en la fase de extinción y $R$ LB se refiere a la tasa de respuesta de la última sesión de línea base previa a la prueba de resistencia. El resultado de la división se transformó en logaritmos.

\section{Resultados}

Para el análisis de los datos, se describen las tendencias en las tasas de respuesta (unir rompecabezas) en cada componente durante la fases del estudio, para los cuatro participantes, la tasa promedio de reforzamiento obtenido, la razón de cambio (en cada componente para cada prueba de resistencia, a partir de la tendencia en las tasas respuestas, en grupos de tres minutos por sesión, con respecto a la última sesión 
de línea base) y los valores percibidos por los participantes sobre la cantidad de reforzamiento obtenido durante las LB, a partir de los cuestionarios.

La Figura 2 representa el número de respuestas por minuto, para los tres componentes del programa durante las fases de LB, NC y EXT, para cada participante. El nivel de respuestas durante las fases de LB para los componentes sin reforzamiento alternativo del lado derecho (asociados con extinción) siempre fue mayor que el nivel de respuestas del componente con reforzamiento alternativo (RDA IV20 IV60). La tendencia de respuesta en LB se mostró estable en las últimas cinco sesiones de las fases para todos los participantes.

Las tendencias de respuesta durante EXT para los componentes sin RDA (EXT IV15 y EXT IV60) disminuyeron para los participantes P1, P2 y P3 y se mantuvieron estables para P4. Las tendencias de respuesta durante $\mathrm{NC}$ para los mismos componentes (sin RDA) decrementaron para P2, P3 y P4 y se mantuvieron estables para P1.

Las tendencias de respuesta durante EXT para el componente con RDA (IV20 IV60) incrementaron para P1 y aumentaron y se mantuvieron en un nivel mayor al de LB para P2, P3 y P4. Las tendencias de respuesta durante NC para el componente con RDA aumentaron para P1, mostraron variabilidad pero en un nivel mayor al de línea base para P2 y P3, y se mantuvieron estables para P4.

En la Figura 3, muestran las tasas de respuesta en proporciones de LB durante la EXT y NC, y se observa que éstas son mayores y positivas en todos los participantes cuando existía RDA (IV20 IV60). Particularmente, el participante P1 no mostró reducción en la tasa de respuesta en las pruebas de resistencia en los componentes sin RDA. Los tres participantes restantes si mostraron un efecto de las pruebas, observándose una disminución en la tasa de los componentes sin RDA y todos los participantes mostraron un efecto de las pruebas de resistencia al cambio en el componente con RDA mostrando un mantenimiento o aumento en su tasa de respuesta.

En términos globales, entonces, la tasa de respuesta promedio del componente EXT IV15 fue similar (29.32 respuestas/minuto) a la del componente EXT IV60 (26.14/ minuto), durante la LB previa a las prueba NC. La tasa promedio de respuesta más baja ocurrió durante el componente con RDA IV20 IV60 (13.44/minuto). La tasa de respuesta promedio para el componente EXT IV15 también fue similar (36.92 respuestas/minuto) a la del componente EXT IV60 (34.49/minuto), durante la LB previa a la EXT. De igual forma, la tasa promedio de respuesta más baja ocurrió durante el componente con RDA IV20 IV60 (10.32/minuto).

El promedio de la tasa de respuesta global en los dos componentes asociados con extinción disminuyó en la prueba no contingente un $5.67 \%$ en promedio en el componente EXT IV15 y un $1.3 \%$ en el EXT IV60 y en extinción un $10.37 \%$ con respecto al promedio de la línea base en el componente EXT IV15 y un $7.59 \%$ en el componente EXT IV60. En las fases de prueba de resistencia al cambio, la tasa de 


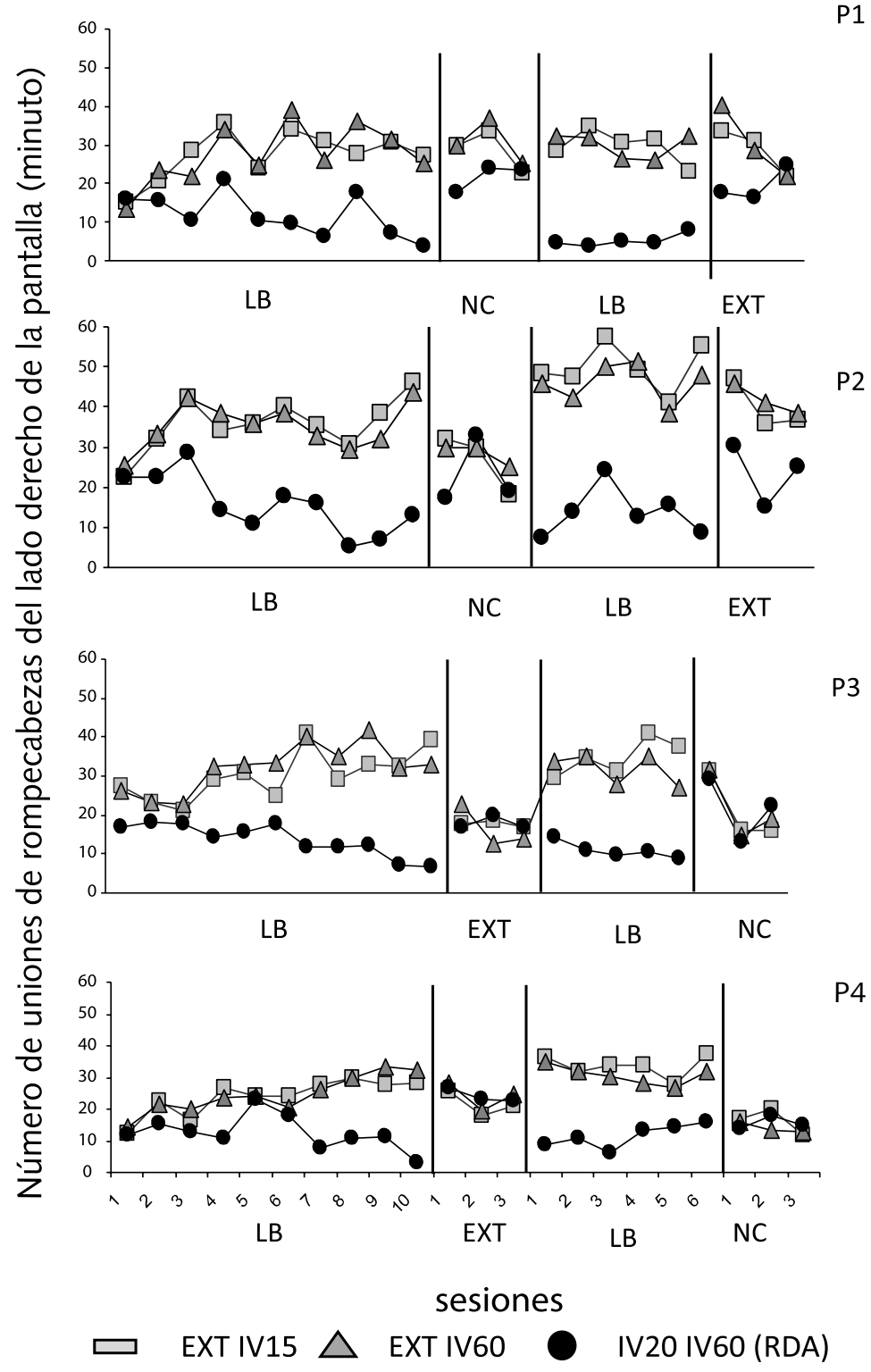

Figura 2. Se representa el número de uniones de rompecabezas por minuto realizadas por el niño del lado derecho de la pantalla por minuto, en cada componentes del programa durante las sesiones de línea base (LB), extinción (EXT) y entrega no contingente del reforzador (NC) para los cuatro participantes. 


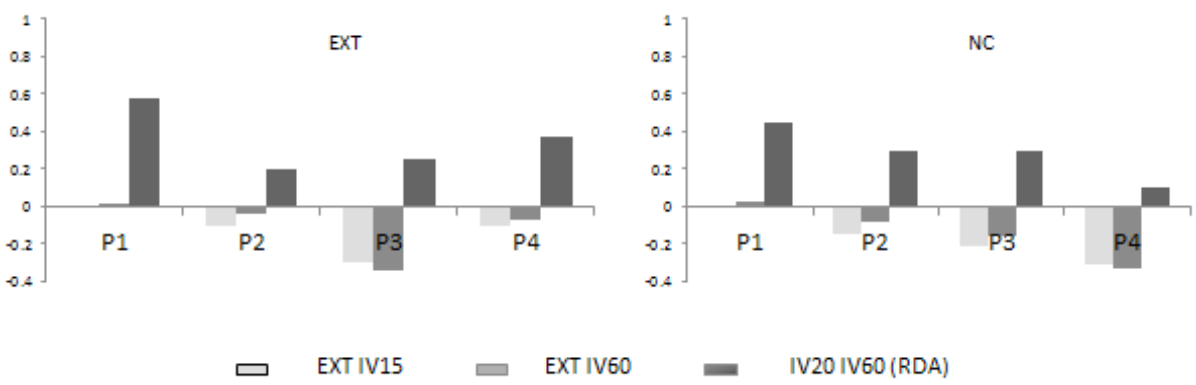

Figura 3. Se representa las tasas durante la EXT y NC como proporciones de línea base para los cuatro participantes.

respuesta del componente con RDA (IV20 IV60) aumentó un $8.20 \%$ en la NC y un 9.67\% en EXT contra el promedio de línea base.

En la Tabla 2 se muestran las tasas promedio de reforzamiento obtenido del lado derecho para cada uno de los componentes del programa múltiple y para los dos lados del componente IV20 IV60 (RDA) durante las dos fases de línea base. La tasa de reforzamiento obtenido resultó acorde a lo programado con una mayor tasa de reforzamiento del lado derecho en el componente EXT IV15, seguida por las tasas de reforzamiento de los componentes EXT IV60 e IV20 IV60 del mismo lado. También se muestra que las tasas promedio globales de reforzamiento del componente EXT IV15 e IV20 IV60 fueron similares, tal como se programó.

Tabla 2

Tasas promedio de reforzamiento obtenido del lado derecho de los componentes del programa múltiple concurrente y global obtenido del componente IV20 IV60 en las líneas base, para la tarea (unir rompecabezas de dos piezas).

\begin{tabular}{ccccc}
\hline & $\begin{array}{c}\text { Lado } \\
\text { derecho } \\
\text { EXT IV15 }\end{array}$ & $\begin{array}{c}\text { Lado } \\
\text { derecho } \\
\text { EXT IV60 }\end{array}$ & $\begin{array}{c}\text { Lado } \\
\text { derecho } \\
\text { IV20 IV60 }\end{array}$ & $\begin{array}{c}\text { Todo el } \\
\text { componente } \\
\text { IV20 IV60 }\end{array}$ \\
\hline $\begin{array}{c}\text { Tasas promedio } \\
\text { de reforzamiento } \\
\text { obtenido }\end{array}$ & 3.53 & 0.99 & 0.8 & 3.63 \\
\hline
\end{tabular}

La Figura 4 representa la razón de cambio graficada (Ecuación 1) en una escala logarítmica, para todos los participantes en las dos fases de prueba. Los puntajes 


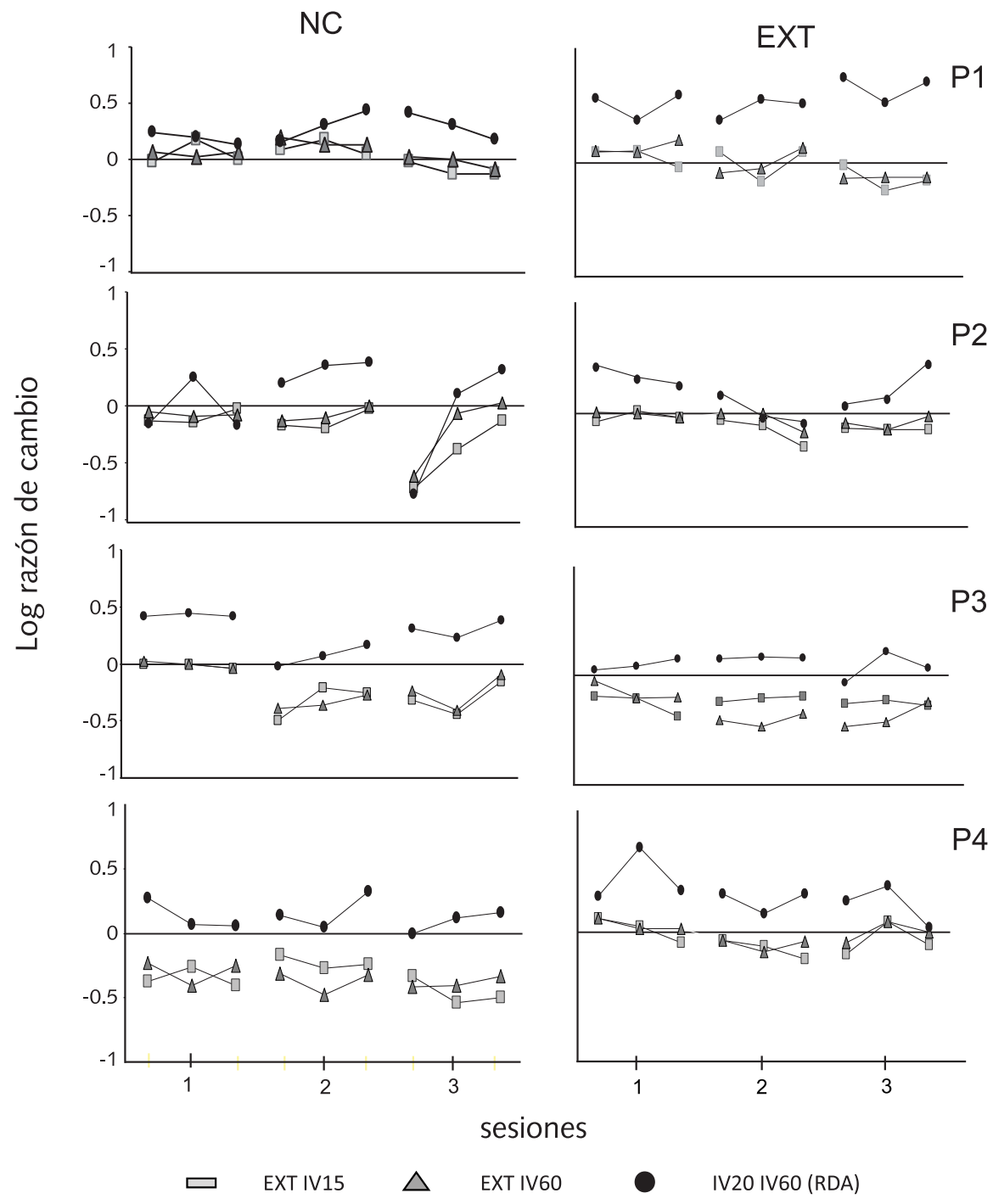

Figura 4. Razones de cambio de la respuesta, en logaritmos, organizadas en bloques de tres minutos por sesión para cada componente del programa. La columna izquierda representa los datos durante la prueba de entrega No contingente del reforzador (NC) y la derecha durante la Extinción (EXT). Cada renglón corresponde a cada participante. Los puntos cercanos a cero representan la ausencia de cambio. 
alrededor de cero representan la ausencia de cambio, mientras que los valores positivos y los negativos representan aumento o disminución en la resistencia al cambio, respectivamente, con relación al nivel exhibido durante la última sesión de LB (de acuerdo al procedimiento de Nevin et al., 1990). La razón de cambio de los componentes sin RDA EXT IV15 y EXT IV60 disminuyó durante las sesiones de NC y EXT para todos los participantes y hasta la última sesión. Las disminuciones en las tasas de respuesta representaron una reducción en su resistencia al cambio. La razón de cambio del componente con RDA IV20 IV60 aumentó, mostrándose sobre cero a lo largo de las sesiones, para los cuatro participantes en ambas pruebas y se mostró reducida solamente para el participante 2, en los primeros 3 minutos de la sesión 3, durante la entrega no contingente del reforzador.

Por último, la Tabla 3 representa los valores percibidos por los niños sobre la cantidad de reforzamiento obtenido durante las líneas base. Los cuatro niños reportaron valores más altos en el componente EXT IV15 del lado derecho seguido por el lado izquierdo del componente IV20 IV60 y con los valores más bajos en los componentes EXT IV60 e IV20 IV60 del lado derecho, todos ellos cercanos al real obtenido.

Tabla 3

Frecuencia de puntos percibidos por los participantes para cada lado en el programa múltiple concurrente utilizado en las fases de línea base.

\begin{tabular}{ccccccc}
\hline & \multicolumn{2}{c}{ EXT IV15 } & \multicolumn{2}{c}{ EXT IV60 } & \multicolumn{2}{c}{ IV20 IV60 } \\
& Izquierda & Derecha & Izquierda & Derecha & Izquierda & Derecha \\
\hline S1 & 0 & 37 & 0 & 9 & 30 & 10 \\
S2 & 0 & 39 & 0 & 9 & 16 & 9 \\
S3 & 0 & 34 & 0 & 9 & 36 & 9 \\
S4 & 0 & 35 & 0 & 9 & 18 & 9 \\
\hline
\end{tabular}

\section{Discusión}

Con el fin de identificar la aplicabilidad de las premisas de la teoría de resistencia al cambio para el posterior tratamiento eficiente de problemas de conducta de relevancia social, el objetivo de este trabajo consistió en describir las variables que determinan la resistencia al cambio de una conducta realizada en computadora con niños en edad escolar a través de situaciones controladas en un escenario de transición, a través de un programa múltiple concurrente (Nevin et al., 1990; Morales \& Santoyo, 2012). 
Los resultados apoyaron dos premisas importantes. Primero, la tasa de respuesta, mantenida por un programa de intervalo variable, fue baja cuando se otorgó reforzamiento simultáneo a otra respuesta alternativa (Mace et al., 1990; Morales \& Santoyo, 2005, 2012; Mace et al., 2010; Nevin et al., 1990; Nevin \& Shahan, 2011). Las tasas de respuesta del componente con RDA IV20 IV60 en las LB se encontraron por debajo de los dos componentes asociados con extinción, (sin RDA). Del mismo modo, los datos apoyaron que la resistencia al cambio fue mayor en este componente con reforzadores adicionales contingentes a la respuesta alternativa (Catania, 1969; Morales \& Santoyo, 2005, 2012; Nevin et al., 1990; Mace et al., 2010; Nevin \& Shahan, 2011). Tanto en la fase NC como en la EXT, las tasas de respuesta para el componente IV20 IV60 se mantuvieron constantes o incrementaron. Cuando los datos se expresaron como proporciones de línea base, la resistencia a la EXT y a NC fue evidentemente mayor en el componente con RDA IV20 IV60 que en los dos componentes restantes (Nevin \& Shahan, 2011). Las razones de cambio obtenidas a partir de la proporción entre las tasas de respuesta durante las fases de prueba con relación a las LB señalan que la tasa de respuesta del componente donde existe reforzamiento a una conducta alternativa es más resistente al cambio, comparada con las razones de cambio de los otros dos componentes.

Los resultados indicaron, entonces, que los reforzadores ante la respuesta con RDA fortalecieron la relación estimulo-reforzador al incrementarse la tasa de reforzamiento recibida en presencia del estímulo en el cual ocurría la respuesta, por lo que se mostró una mayor resistencia al cambio en el componente con RDA IV20 IV60. La reducción en la tasa de respuesta de los componentes sin RDA, ocurrió paulatinamente y se hicieron evidentes a lo largo de las sesiones y hasta la tercera sesión de prueba para todos los participantes, por lo que el efecto de la disrupción por las pruebas de resistencia fue lento y progresivo. Al final se observó una menor resistencia al cambio en los componentes sin RDA.

El componente EXT IV60, mostró una baja resistencia al cambio, tal como se esperaba. Sin embargo la resistencia al cambio también fue baja para el componente EXT IV15, que otorgó reforzamiento global similar al componente con RDA. Los datos parecen indicar que el RDA si incrementa la tasa global de reforzamiento pero también genera un efecto parcial de extinción (D'Amato, Lachman, \& Kivy, 1958; Mandell, 2000). Nevin y Shahan (2011) señalaron que la extinción y el reforzamiento NC suspenden la contingencia entre la respuesta y el reforzador, reduciéndose el responder debido al decremento en la generalización y al incremento tanto del efecto de la suspensión de la contingencia como del decremento en la generalización al pasar del tiempo. Con base en la hipótesis del decremento en la generalización (Capaldi, 1967; Nevin \& Grace, 2000) el número de respuestas sin 
recibir reforzamiento en LB, funcionan como parte del estímulo discriminativo asociado al color que señala la posibilidad de reforzamiento próximo. Así, los organismos responden con mayor o menor fuerza durante la EXT o NC, dependiendo de qué tan similar sea el estímulo de prueba al estímulo original de entrenamiento. En este sentido, Nevin y Grace (2000) demostraron que el efecto parcial de extinción se encuentra presente en los estudios de resistencia al cambio. Al extender el modelo sobre resistencia a la extinción, los autores concluyeron que cuando la tasa de reforzamiento es muy elevada, la tasa de respuesta decae conforme incrementa aún más la tasa de reforzamiento, pues el estímulo asociado a las respuestas durante la fase de prueba llega a ser muy diferente en los programas con altas tasas de reforzamiento de aquellos con tasas más bajas.

La extinción ocurre rápidamente cuando la omisión del reforzador es altamente notoria. La curva de extinción se revierte cuando la omisión del reforzador es menos evidente (Nevin \& Shahan, 2011). La tasa de respuesta no tuvo consecuencias durante EXT y los reforzadores también se otorgaron de manera independiente a la respuesta en NC como en la LB, por lo que el efecto de la suspensión de la contingencia para el componente IV20 IV60, pareció ser menos perceptible que en EXT IV15, que otorgó el mismo reforzamiento global, pero cuyos estímulos del contexto se mostraron diferentes. Nevin y Shahan (2011) refieren que la presentación de reforzamiento no contingente podría reducir el efecto de la prueba de resistencia porque el ambiente incluye reforzadores, provocando una tasa de respuesta prolongada durante la fase, tal como se observó en el componente con RDA, y aunque el componente EXT IV15 entregó reforzamiento global similar, el reforzamiento asociado al estímulo discriminativo fue diferente al del componente IV20 IV60. En los estímulos discriminativos asociados al color de las fichas en el componente EXT IV15 se observaron tiempos cortos entre respuestas, mientras que los estímulos que controlaron las tasas de respuesta en las pruebas durante el componente IV20 IV60 estuvieran constituidos por tiempos entre respuestas mayores (Nevin \& Shahan, 2011). Es posible que el decaimiento en la tasa de respuesta en el componente EXT IV15 fuera similar al efecto parcial en extinción observado por Nevin y Grace (2000) y que no solo la tasa de reforzamiento, sino el decremento en la generalización de los estímulos, para estos participantes, fueran los determinantes de la baja tasa de respuestas durante las pruebas; mientras que en el componente IV20 IV60, el efecto de la situación combinado con el efecto de suspender la contingencia fuera menor (Nevin \& Shahan, 2011).

Así, el efecto de retirar el reforzamiento puede ser imperceptible en ambientes aplicados, donde se utiliza el elogio durante la intervención y la extinción. Sin un cambio en el ambiente o en la conducta del terapeuta, se obtendría un efecto parcial de extinción. En el ámbito aplicado se utiliza el reforzamiento de conducta alternativa, 
cuando los problemas de conducta no se pueden reducir solo por medio de la extinción ya sea porque empeoran (agresión) o porque no se puede retirar el reforzamiento (auto-estimulación). El reforzamiento diferencial de conducta alternativa (RDA) es altamente efectivo (Morales \& Vázquez, 2011; Morales, 2012); sin embargo, la investigación básica lo ha usado para reducir las tasas de respuesta en línea base, provocando, paradójicamente, una mayor resistencia al cambio. Agregar reforzadores en el contexto de estímulos discriminativos incrementa la resistencia a la extinción y agregar reforzadores independientes a la respuesta incrementa su resistencia al cambio (Nevin \& Shahan, 2011).

Mace et al. (2010) también utilizaron contingencias RDA para reducir conductas problema y encontraron que la conducta meta decremento más rápidamente y a un nivel más bajo cuando se aplicó extinción directamente después de la LB que cuando se aplicó extinción después del tratamiento con RDA. La tasa de respuesta del componente RDA IV20 IV60 pudo mostrar la misma tendencia que señala Mace et al. (2010), de reducirse más lentamente en las fases de prueba. En este sentido, Nevin y Shahan (2011) indicaron que la tasa de la conducta problema puede resurgir si primero es reducida por medio del RDA y luego extinguida, que si extingue directamente sin RDA, como fue el caso de los componentes sin RDA, EXT IV15 y EXT IV60, cuyas tasas se redujeron más eficientemente que las del RDA en IV20 IV60. En el ámbito aplicado, el resurgimiento de la conducta meta significa que la terminación del RDA, puede generar la reaparición de la conducta problema después de una intervención, reflejando la importancia del comprometerse con la integridad del tratamiento. La magnitud del resurgimiento será mayor con tasas altas de RDA y por lo tanto debe considerarse la posibilidad de este efecto colateral contra-terapéutico cuando se implementen programas de RDA de tasa alta (Nevin \& Shahan, 2011).

En continuidad con los hallazgos del estudio, al no encontrar resistencia al cambio similar entre el componente EXT IV15 y aquel con RDA IV20 IV60 en 6 de 8 pruebas, ya que solo P3 durante EXT y P4 durante NC mostraron tasas de respuesta similares entre los componentes con tasa global de reforzamiento (Cohen, 1986; Cohen et al., 1993; Harper \& McLean, 1992; Leslie, 2000; Morales \& Santoyo, 2005, 2012), el presente estudio requiere mayor evidencia para afirmar que exista independencia entre los determinantes de las tasas de respuesta (relación respuesta-reforzador) y aquellos de la resistencia al cambio (relación estímulo-reforzador), aun cuando, si se observa un efecto parcial de extinción por decremento en la generalización de estímulos, obteniendo mayor resistencia al cambio en el componente con RDA (Nevin \& Shahan, 2011).

Una alternativa viable para comprender la diferencia en las tasas de respuesta durante las pruebas de resistencia en los dos programas con tasas globales de refor- 
zamiento similares es que en los dos componentes asociados con extinción: EXT IV15 y EXT IV60, las tasas de respuesta también fueron similares durante las LB, a pesar de que el primer componente proporcionó cuatro veces más reforzamiento que el segundo, hallazgo consistente con lo obtenido por Mace et al. (1990) y por Morales y Santoyo $(2005,2012)$. Weiner (1983) y Hyten y Madden (1993) reportaron que los participantes humanos incrementan ineficazmente el número de respuestas, excediendo el mínimo requerido para obtener el mayor reforzamiento disponible. Es posible que los participantes a lo largo del estudio emitieran más respuestas de las requeridas en el componente EXT IV60, por lo que su nivel de conducta se mostró equivalente al del componente EXT IV15, mostrando una posible baja sensibilidad a los programas de intervalo variable. De acuerdo con Matthews, Shimoff, Catania \& Sagvolden (1977) los seres humanos tienden a mostrar baja sensibilidad a los programas de intervalo variable debido a las instrucciones o al efecto de la ausencia del consumo inmediato del reforzador, característica que si ocurre en pichones y ratas.

Con relación a si las instrucciones son variables determinantes de la sensibilidad de los humanos a los IV, es importante señalar que en este estudio, los cuestionarios resueltos por los participantes permitieron identificar que los participantes fueron sensibles a los programas de intervalo variable. Aunque los investigadores no utilizaron las instrucciones intencionalmente para moldear el responder como Matthews et al. (1977) sugieren para los estudios con humanos, el entrenamiento previo tuvo el objetivo de generar tasas de respuesta similares para ambos lados de los componentes del programa múltiple concurrente. Las respuestas verbales de los participantes sobre el reforzamiento obtenido percibido, indican que dedujeron la regla de reforzamiento. Por lo que puede atribuirse sensibilidad de los participantes a los valores de los programas de IV.

En cuanto al efecto del consumo del reforzador es importante indicar que, aunque en el presente estudio el reforzador no era consumible, si existían pausas de respuesta ante la entrega de puntos con fichas, acompañadas del sonido. La presentación del sonido de entrega del reforzador, así como la entrega de la ficha en la mesa del participante interrumpían el patrón de respuesta y aunque no se midió si representaban un "costo físico del responder" (Weiner, 1962, p. 6) no se puede afirmar que las respuestas fueran representativas de insensibilidad a los programas de intervalo. Adicionalmente, considérese que el nivel de disrupción de las pruebas aunque paulatino fue efectivo en este estudio.

No obstante que se pudiera considerar que los participantes fueron sensibles a los programas IV, en futuros estudios se recomienda agregar un costo por el acceso al reforzador para conocer el grado de mejora en la eficiencia de la interrupción de la prueba (Weiner, 1962). Así mismo, existe la posibilidad de que las respuestas 
emitidas durante las fases de LB y las de prueba pudieran considerarse "erráticas" (Weiner, 1962, p. 4) de tipo exploratorio, donde se requiriera un estímulo discriminativo que interrumpiera el patrón de conducta para mostrarse como característico del programa de intervalo variable de un componente particular y diferencial al resto de los componentes. También es probable que las tendencias de los patrones de respuesta establecidas en línea base representaran la generalización o imitación de respuestas durante las fases de prueba entre los componentes asociados con extinción y que este efecto, combinado con el del cambio en las contingencias y en la situación de estímulos, explicara la similitud en la resistencia al cambio en los dos componentes con tasa diferencial de reforzamiento y la razón de cambio desigual en los dos componentes con tasa equivalente de reforzamiento (Morales \& Santoyo, 2005, 2012).

Otras explicaciones de la resistencia diferencial al cambio en dos programas con una tasa similar de reforzamiento global, se refiere a la topografía de la respuesta. Baum y Mitchell (2000) afirmaron que la teoría de la resistencia al cambio, tal como Nevin y Grace (2000) la sustentaron, no contempla la topografía de la respuesta y que esta puede ser determinante en la explicación de la resistencia al cambio. De acuerdo con Galbicka y Kessel (2000) la energía de una respuesta se modifica con cada interacción y que una respuesta previa es diferente a la que le sigue. Sin embargo, aún no se tiene evidencia empírica que señale el grado en que la topografía de la respuesta o la interacción entre las respuestas o el medio afecten su propia resistencia al cambio. En esta investigación, la topografía de la conducta no parece haber generado efectos sustanciales en los hallazgos sobre la resistencia al cambio. Se programó que las conductas fueran operantes discriminadas observando una elevada tasa de respuesta (Santoyo, 2012) controlando el tiempo de exposición a la variable independiente hasta lograr estabilidad en la conducta meta (Baron, Perone, \& Galizio, 1991; Matthews, Shimoff, Catania, \& Sagvolden, 1977; Shull \& Lawrence, 1998), tanto en el pre-entrenamiento como en las diversas líneas base, para controlar su historia de reforzamiento. Así, tanto las respuestas como los reforzadores fueron funcionalmente representativos del fenómeno a estudiar y análogos a los utilizados en estudios previos con sujetos no humanos.

Adicionalmente, el uso de reforzamiento condicionado representa una explicación alternativa a los resultados de esta investigación. La similitud entre las tasas de respuesta entre los componentes EXT IV15 Y EXT IV60, puede deberse a la tendencia de los humanos a responder indiscriminadamente ante programas de IV diferentes cuando se utiliza reforzamiento condicionado (Fisher \& Mazur, 1997). Aparentemente, el tiempo entre la entrega del reforzamiento condicionado y el acceso al reforzamiento "primario" afecta directamente la tasa de respuesta durante los programas de reforzamiento. En este estudio y a través de los cuestio- 
narios, se evaluó el valor percibido del reforzador. Todos los niños reportaron ganancias percibidas similares a las obtenidas y programadas. De acuerdo con Rachlin (1976) los niños responden con tasas de respuestas elevadas, aparentemente para asegurar sus ganancias, cuando se utiliza este tipo de reforzamiento. Es posible que esta tendencia en el responder durante las líneas base afecte también la resistencia al cambio obtenida del componente EXT IV15, a pesar de proporcionar una tasa global de reforzamiento similar que el componente con RDA IV20 IV60.

En conclusión, los resultados permiten afirmar que la relación respuesta-reforzador determinó parcialmente la tasa de respuesta de unir rompecabezas, al debilitar dicha relación a través de reforzamiento adicional a la conducta alternativa en el mismo componente, y que la relación estímulo-reforzador determinó parcialmente la resistencia al cambio de la conducta medida, al observar una tasa de respuesta constante durante la fases de prueba para ese mismo componente. El Reforzamiento a una conducta alternativa redujo su tasa de respuesta durante la LB y decremento su resistencia al cambio a la EXT y NC. Sin embargo, se requiere mayor sustento empírico para probar la independencia entre los determinantes de la resistencia al cambio. Es decir, la resistencia al cambio estuvo determinada parcialmente por la relación estímulo-reforzador establecida aquí y por un efecto parcial en extinción en el componente con RDA IV20 IV60, donde la tasa de reforzamiento a una conducta alternativa pero global elevada en un contexto particular produjo una menor tasa de respuesta durante la LB y un efecto del decremento en la generalización de estímulos produjo una mayor tasa de respuesta durante las pruebas de resistencia al cambio (Morales \& Santoyo, 2012; Nevin \& Shahan, 2012).

En el presente estudio se reforzó la conducta de unir rompecabezas de dos piezas y se identificó el papel relativo de la relación estímulo-reforzador para determinar parcialmente su resistencia al cambio. El estudio aporta y contribuye información sobre la naturaleza del principio de resistencia al cambio con humanos. La investigación traslacional es una opción que requiere que verifiquemos si se cumple la extensión de los principios y su potencial eficiencia en situaciones de la vida cotidiana. En el caso de las intervenciones para la eliminación de la conducta disruptiva en niños, donde por décadas se ha aplicado el procedimiento de reforzamiento diferencial de conducta alternativa a la conducta meta, la identificación de aquellas condiciones contextuales que producen mayor persistencia del comportamiento, puede ahorrar complicaciones en el establecimiento y mantenimiento de conducta socialmente aceptables, durante y después de las intervenciones conductuales instrumentadas en el campo de la Psicología traslacional, (Morales, 2012) evitando el resurgimiento de la conducta meta de tratamiento (Nevin \& Sh- 
ahan, 2011) y promoviendo el compromiso de los profesionales con la programación del mantenimiento de los cambios al concluir las intervenciones en el ámbito clínico.

La teoría de resistencia al cambio establece que los reforzadores incrementan la persistencia de la conducta frente a los retos o las disrupciones tales como la distracción o la eliminación del reforzamiento. También establece que la conducta meta en una situación dada incrementa por los reforzadores alternativos otorgados en esa situación, sin importar si son contingentes a una respuesta alternativa. El modelo puede servir como una guía para la intervención cuando se identifican sus términos con las condiciones y contingencias de los escenarios aplicados (Nevin y Shahan, 2011). La investigación traslacional debe usarse como preparación para la identificación de las variables controladoras de comportamientos focales de relevancia social (Santoyo 2012). Este trabajo pretendió aportar acciones concretas para el desarrollo de principios básicos y estrategias metodológicas que redirijan la atención hacia problemas de relevancia social y de la vida cotidiana.

\section{Referencias}

Baron, A., Perone, M., \& Galizio, M. (1991). Analyzing the reinforcement process at the human level: Can application and behaviorist interpretation replace laboratory research? The Behavior Analyst, 14, 95-105.

Baum, W. M., \& Mitchell, S. H. (2000). Newton and Darwin: Can this marriage be saved? Behavioral and Brain Sciences, 23, 91-92.

Buskist, W., Morgan, D., \& Terrell, D. J. (1985). On the experimental analysis of human behavior. Revista Mexicana de Análisis de la Conducta, 11, 69-78.

Capaldi, E. J. A. (1967). Sequential hypothesis of instrumental learning. In K. W. Spence y J. T. Spence (Eds.). The Psychology of Learning and Motivation, 1, (pp. 381). New York: Academic Press.

Catania, A. C. (1969). Concurrent performances: Inhibition of one response by reinforcement of another. Journal of the Experimental Analysis of Behavior, 12, 731-744.

Cohen, S. L. (1986). A pharmacological examination of the resistance to change hypothesis of response strength. Journal of the Experimental Analysis of Behavior, 46, 363-379.

Cohen, S. L., Riley, D. S., \& Weigle, P. A. (1993). Tests of behavior momentum in simple and multiple schedules with rats and pigeons. Journal of the Experimental Analysis of Behavior, 60, 255-291.

D'Amato, M. R., Lachman, R., \& Kivy, P. (1958). Secondary reinforcement as affected by reward schedule and the testing situation. Journal of Comparative and Physiological Psychology, 51, 734-741. 
Fisher, W. W., \& Mazur, J. E. (1997). Basic and applied research on choice responding. Journal of Applied Behavior Analysis, 30, 387-410.

Fleshler, M., \& Hoffman, H. S. (1962). A progression for generating variable-interval schedules. Journal of the Experimental Analysis of Behavior, 5, 529-530.

Galbicka, G., \& Kessel, R. (2000). Experimenter momentum and the effect of laws. Behavioral and Brain Sciences, 23, 97-98.

Harper, D. N., \& McLean A. P. (1992). Resistance to change and the law of effect. Journal of the experimental analysis of behavior, 57, 317-337.

Harper, D. N. (1999). Drug induced changes in responding are dependent upon baseline stimulus-reinforcer contingencies. Psychobiology, 27, 95-104.

Herrnstein, R. J. (1970). On the law of effect. Journal of the Experimental Analysis of Behavior, 13, 243-266.

Houlihan, D., \& Brandon, P. K. (1996). Compliant in a Moment: A commentary on Nevin. Journal of Applied Behavior Analysis, 29, 549-555.

Hyten. C., \& Madden, G. J. (1993). The scallop in human fixed-interval research: A review of problems with data description. Psychological Record, 43, 471-500.

Leslie, J. C. (2000). Does conditioned suppression measure the resistance to change of operant behavior? Behavioral and Brain Sciences, 23, 103-104.

Mace, F. C., \& Belfiore, P. (1990). Behavioral momentum in the treatment of escapemotivated stereotypy. Journal of Applied Behavior Analysis, 23, 507-514.

Mace, F. C., Hock, M. L., Lalli, J. S., West, B. J., Belfiore, P., Pinter, E., \& Brown, D. K. (1988). Behavioral momentum in the treatment of noncompliance. Journal of Applied Behavior Analysis, 21, 123-141.

Mace, F. C., Lally, J. S., Shea, M. C., Lalli, E. P., West, B. J., Roberts, M. \& Nevin, J. A. (1990). The momentum of human behavior in a natural setting. Journal of the Experimental Analysis of Behavior, 54, 163-172.

Mace, F.C., McComas, J. J., Mauro, B. C., Progar, P. R., Taylor, B., Ervin, R., \& Zangrillo, A. N. (2010). Differential reinforcement of alternative behavior increases resistance to extinction: Clinical demonstration, animal modeling, and clinical test of one solution. Journal of the Experimental Analysis of Behavior, 93 (3), 349-367.

Mandell, C. (2000). The partial reinforcement effect and behavioral momentum: reconcilable? Behavioral and Brain Sciences, 23, 106-107.

Matthews, B. A., Shimoff, E., Catania, A. C., \& Sagvolden, T. (1977). Uninstructed human responding: Sensitivity to ratio and interval contingencies. Journal of the Experimental Analysis of Behavior, 27, 453-467.

Mauro, B. C., \& Mace, F. C. (1996). Differences in the effect of Pavlovian contingencies upon behavioral momentum using auditory versus visual stimuli. Journal of Experimental Analysis of Behavior, 65, 389-399.

Mcllvane, W. J., \& Dube, W. V. (2000). Behavioral momentum and multiple stimulus 
control topographies. Behavioral and Brain Sciences, 23, 109.

Morales, C. S. (2012). Articulo comentario a artículo A Investigación traslacional: Una misión prospectiva para la Ciencia del Desarrollo y la Ciencia del Comportamiento. Revista Mexicana de Investigación en Psicología, 4(2), 111.

Morales, C. S. (2012). Estilos de Crianza Positiva para prevenir el consumo de alcohol en menores de edad. En R.L. García \& M.B. Barriguete (Eds). Consumo de A/cohol y Tabaco en México (pp. 88-111). México: Secretaría de Salud. 291. ISBN: 978607-95887-3-1.

Morales, C. S., \& Santoyo, V. C. (2005). Evaluación de la resistencia al cambio de la conducta en niños. Revista Mexicana de Psicología, 22(1),177-192.

Morales, C. S. \& Santoyo, V. C. (2012). Resistencia al cambio de una conducta académica en niños. Revista Mexicana de Análisis de la Conducta, 38(1), 39-60.

Morales, C. S., \& Vázquez, P. F. (2011). Evaluación de conocimientos sobre habilidades de manejo conductual infantil en profesionales de la salud. Acta de Investigación Psicológica, 1(3), 428-440.

Nevin, J. A., \& Grace, R. C. (2000). Behavioral momentum and the Law of Effect. Behavioral and Brain Sciences, 23, 73-130.

Nevin, J. A., \& Shahan, T. A. (2011). Behavioral Momentum Theory: Equations and Applications. Journal of Applied Behavior Analysis, 44 (4), 877-895.

Nevin, J. A. (1974). On the form of the relation between response rates in a multiple schedule. Journal of the Experimental Analysis of Behavior, 21, 237-248.

Nevin, J. A. (1979). Reinforcement schedules and response strength. In M. Zeiler \& P. Harzem (Eds.), Reinforcement and organization of behavior (pp. 117-158). New York: John Wiley \& Sons.

Nevin, J. A. (1996). The momentum of compliance. Journal of Applied Behavior Analysis, 29, 535-457.

Nevin, J. A., Mandell, C., \& Atak, J.R. (1983). The analysis of behavioral momentum. Journal of the Experimental Analysis of Behavior, 39, 49-59.

Nevin, J.A., Tota, M.E., Torquato, R.D., \& Shull, R.L. (1990). Alternative reinforcement increases resistance to change: Pavlovian or operant contingencies? Journal of the Experimental Analysis of Behavior, 53, 359-379.

Parry-Cruwys, D. E., Neal, C. M., Ahearn, W. H., Wheeler, E. E., Presmchander, R., Loeb M. B. \& Dube, W. V. (2011). Resistance to disruption in a classroom setting. Journal of Applied Behavior Analysis, 44(2), 363-367.

Rachlin, H. C. (1976). Behavior and Learning. Sn Francisco: Freeman and Company. 545-589.

Roane, H. S., Kelley, M. E., Trosclair, N. M., \& Hauer, L. S. (2004). Behavioral momentum in sports: A Partial Replication with women's basketball, Journal of Applied Behavior Analysis, 37(3), 385 - 390. 
Santoyo, V. C. (2012). Investigación traslacional: Una misión prospectiva para la Ciencia del Desarrollo y la Ciencia del Comportamiento, Revista Mexicana de Investigación en Psicología, 4(2),84-110.

Santoyo, V. C., Morales, C. S., Colmenares, V. L., \& Figueroa, N (2007). Organización del comportamiento en el aula: transiciones, persistencia, interrupciones e interferencia social. En C. Santoyo (Ed), Estabilidad y cambio de patrones de comportamiento en escenarios naturales: Un estudio longitudinal en Coyoacán (pp. 181-206). México, D.F.: UNAM-CONACYT.

Shull, R. L., \& Lawrence, S. (1998). Reinforcement Schedule Performance. In K. A. Lattal \& M. Perone, (Eds), Handbook of Research Methods in Human Operant Behavior (pp. 95-127). New York: Plenum Press.

Weiner, H. (1962). Some effects of response cost upon human operant behavior. Journal of the Experimental Analysis of Behavior, 5(2), 201-208.

Weiner, H. (1983). Some thoughts on discrepant human-animal performances under schedules of reinforcment. The Psychological Record, 33, 521-532. 